\title{
Effect of a Delta Tab on Fine Scale Mixing in a Turbulent Two-Stream Shear Layer
}

J.K. Foss and K.B.M.Q. Zaman

Lewis Research Center

Cleveland, Ohio

Prepared for the

34th Aerospace Sciences Meeting and Exhibit

sponsored by the American Institute of Aeronautics and Astronautics

Reno, Nevada, January 15-18, 1996

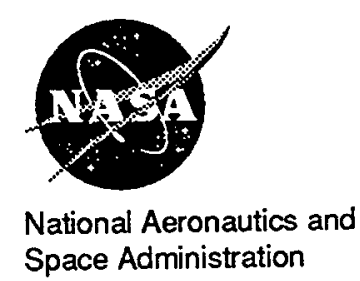




\title{
EFFECT OF A DELTA TAB ON FINE SCALE MIXING IN A TURBULENT TWO-STREAM SHEAR LAYER
}

\author{
J.K. Foss ${ }^{*}$ and K.B.M.Q. Zaman ${ }^{\dagger}$ \\ NASA Lewis Research Center \\ Cleveland, OH 44135
}

\begin{abstract}
$\underline{\text { Abstract }}$
The fine scale mixing produced by a delta tab in a shear layer has been studied experimentally. The tab was placed at the trailing edge of a splitter plate which produced a turbulent two-stream mixing layer. The tab apex tilted downstream and into the high speed stream. Hotwire measurements in the 3-D space behind the tab detailed the three velocity components as well as the small scale population distributions. These small scale eddies, which represent the peak in the dissipation spectrum, were identified and counted using the Peak-ValleyCounting technique. It was found that the small scale populations were greater in the shear region behind the tab, with the greatest increase occurring where the shear layer underwent a sharp turn. This location was near, but not coincident with, the core of the streamwise vortex, and away from the region exhibiting maximum turbulence intensity. Moreover, the tab increased the most probable frequency and strain rate of the small scales. It made the small scales smaller and more energetic.
\end{abstract}

\section{Introduction}

Mixing enhancement has many technological benefits including increased combustion efficiencies and noise reduction. To achieve this via passive control is more desirable than active control in most applications. Intelligent and direct manipulation of the vortex dynamics seems to be a very effective way to increase mixing. Manipulation of the azimuthal vorticity shed from a jet exit can be achieved by using noncircular nozzle shapes. Ho \& Gutmark demonstrated greater than a $100 \%$ increase in mass entrainment using a 2:1 elliptic instead of circular nozzle. The self-induction of azimuthal vortical structures in a noncircular jet usually causes axisswitching and thereby a more efficient engulfment of ambient fluid. ${ }^{1-3}$

Manipulation or production of streamwise vorticity is another effective way to increase entrainment. ${ }^{4,5}$ In a comparative study of the flow fields from various asym-

\footnotetext{
* Research Associate, NRC

${ }^{\dagger}$ Aerospace Engineer, Member AIAA
}

metric nozzle geometries, Zaman ${ }^{3}$ found greater entrainments with geometries enhanced by tabs. A tab is a small protrusion placed into the flow at the jet exit. It generates a pair of counter-rotating streamwise vortices. The dominant source of the streamwise vorticity generated by the tab is an upstream "pressure hill" rather than the reorientation of the boundary layer. ${ }^{4}$ In the case where the tip of the tab leans downstream and into the flow direction, the sense of rotation of the vortex pair is to sweep flow from the base toward the tip. This rotation is opposite to that of the standard boundary layer 'horseshoe' vortex pair. One must use the tab with an understanding of these mechanisms, or else it might not only prove ineffective but even detrimental in certain cases.

Thus far, the tab has demonstrated effectiveness in large scale mixing or stirring, producing an overall increase in entrainment. The large scale mixing, however, is only the first step for most applications. In reacting flows, for example, mixing at the small scales is essential to allow the molecules to combine effectively. In jet engine applications, more efficient small scale mixing is expected to reduce noise via elimination of "hot spots" as well as by shifting the energy to higher frequency bands. Thus, a knowledge of the effect of the tab on small scale mixing is quite critical, and this provided motivation for the present study.

\section{Peak-Valley-Counting Technique}

Until recently, technical limitations have precluded the detailed study of the scale of the structure which dissipates most of the energy. Improvements in measurement and computer technology, however, have made these scales the target of recent focus. ${ }^{6-9}$ In these works, the Peak-Valley-Counting (PVC) technique was used to identify small scale structures directly from the velocity trace. Ho \& $\mathrm{Zohar}^{7}$ have demonstrated that the frequency identified by this technique corresponds to the peak in the 3-D dissipation spectrum.

Application of the PVC technique requires sufficient frequency response and spatial resolution of the sensor, and sampling rates fast enough to describe the scale of interest. This method constructs a pulse train identifying the local peaks and valleys in the velocity 
trace. These extrema are the signatures of the small scale eddies. The method steps through five filtering sequences to distinguish between flow structure and noise. The end result of this yields greater than $90 \%$ accuracy, as determined by human eye.

Once such a pulse train has been established, several diagnostics may be performed. Figure 1a depicts a sample velocity trace and pulse train. Simply counting the number of pulses indicates the number of small scale structures, or population $N_{s s}$, at a point. Since the pulse train retains the temporal information, quantities involving the fine scale period are discernible. A histogram of the inverse period yields a fairly sharp distribution centered around a 'most probable frequency'. It is significant to note that this peak frequency matches that of the dissipation spectrum shown in figure $1 \mathrm{c}$. This $1-\mathrm{D}$ pseudo-dissipation spectrum is defined as $D\left(\kappa_{1}\right)=$ $2 v \int \kappa_{1}^{2} F_{11}\left(\kappa_{1}\right) d \kappa_{1}$, where $\kappa_{1}=2 \pi f / \bar{U}, f$ is the frequency, $\bar{U}$ is the mean velocity, and $F_{11}$ is the $1-D$ energy spectrum. The most probable scale of the eddy detected by the PVC code, then, shall be referred to as the "dissipative eddy". Another analysis to be presented in this study utilizes the amplitude in addition to the temporal information. That is, via Taylor's hypothesis, a measure of the dissipative eddy strain rate, $\Delta u / \Delta x$, is also determined.

Using the PVC technique in a study of an initially laminar, 2-D, two-stream mixing layer, Huang \& $\mathrm{Ho}^{6}$ found that the dissipative eddies first appeared with the first vortex merging location. Moreover, they were not uniformly distributed in the span, but were concentrated near the cores of the streamwise vortices. Furthermore, it was found that while the total number of small scale structures continuously increased downstream, their production rate peaked at the second vortex merging location. ${ }^{7}$ It was conjectured that the increased strain rate, which occurs with the interaction of the vortical structures with each other (spanwise-spanwise or spanwise-streamwise vorticity), generates the small scale structures.

As stated before, a tab has been known to increase overall spreading of a mixing layer. However, because of the complexity of the three dimensional flow field, the details of the large scale vorticity dynamics has remained far from completely clear. On the other hand, only a few previous studies attempted to address the effect of similar passive flow control devices on chemical reaction and the issues of 'mixedness' and small scale mixing. ${ }^{10,11}$ Of note is the work of Zhang \& Schneider, which showed that an increase in overall jet spreading in their study was accompanied by an increase in the product formation. However, it is fair to say that the detailed dynamics of the small scale flow structures, for the tech- nologically important flow field under consideration, has remained virtually unexplored. The PVC code provided an opportunity to explore these details, and this has been the primary objective of the present study.

\section{Experimental Facility and Procedure}

The flow field chosen for the study was a two-stream plane mixing layer. This was generated in a $51 \mathrm{~cm} \times 76$ $\mathrm{cm}$ low speed wind tunnel. ${ }^{12}$ The top speed of this open return, subatmospheric wind tunnel is about $15 \mathrm{~m} / \mathrm{s}$ and the turbulence intensity is less than $0.1 \%$. To generate a two stream mixing layer for this experiment, the following modification was made. A $33 \mathrm{~cm}$ chord symmetric splitter plate spanned the tunnel at mid-height; its maximum thickness was $1.27 \mathrm{~cm}$, and it tapered uniformly down to $0.05 \mathrm{~cm}$. This plate sat on a frame which also housed two 16 mesh screens; the resulting velocity ratio was nominally $2: 1$. Through detailed diagnostic experiments conducted initially, a flap was placed at the leading edge to avoid laminar separation on the upper surface.

The high speed velocity was fixed at $U_{I}=9.32 \mathrm{~m} / \mathrm{s}$, and the low speed velocity at $U_{2}=4.55 \mathrm{~m} / \mathrm{s}$. The shear velocity, $\Delta U=U_{1}-U_{2}$, was used for data normalization. The boundary layers at the plate trailing edge were nominally turbulent, with no identifiable peak in the velocity spectra. The boundary layer thicknesses at the trailing edge were $1.22 \mathrm{~cm}$ and $0.71 \mathrm{~cm}$ for the high and low speed sides, respectively. The shape factors were 1.5 and 2.0, and the momentum thickness Reynolds numbers were $\operatorname{Re}_{\theta}=932$ and 193, for the high and low speed sides, respectively.

The delta tab was a triangular piece of $0.05 \mathrm{~cm}$ thick metal shim, with a $90^{\circ}$ angle at the apex. With the base fixed to the splitter plate trailing edge, the apex tilted into the high speed side at $45^{\circ}$ with respect to the free stream direction. The base of the tab was $2.54 \mathrm{~cm}$ wide and its height was $1.27 \mathrm{~cm}$. Thus, the projected height was $0.90 \mathrm{~cm}$. The tab height and boundary layer thickness were comparable. This was deemed sufficient to produce the expected mixing layer distortion as observed in previous studies. The characteristic length scale for data normalization was chosen to be the tab width, $w$. This choice was based on the finding that the width of the tab is more crucial to the resulting flow field than the height. ${ }^{4}$

A 3-D computer controlled traversing mechanism was used to move the hot-wire probes. The coordinate origin was at the trailing edge at midspan. The trailing edge, the tab, the coordinate system and the data planes are shown in figure 2. The probe was traversed in the cross-stream $(Y, Z)$ plane at several downstream loca- 
tions, $X=0.5,1.0,2.0,4.0,8.5$. The data taken in the transverse direction, $Y$, extended into the potential flow regions on either side of the shear layer. The spatial resolution varied along a profile, but reached a minimum of $\Delta Y=0.1$ in the shear region. The measurements in the spanwise direction, $Z$, are as indicated in figure $2 b$. They extended to $Z=2.0$, where the tab had no effect within the measurement volume, and included one point, $Z=$ -0.25 , on the other side of the symmetry plane. The resolution was $\Delta Z=0.25$ around the tab. For the no tab case, measurements were taken at midspan.

The measurements were conducted using standard hot-wire anemometry. The small scale data were taken with a single straight wire; the secondary velocity and streamwise vorticity measurements were carried out with an X-wire. The single hot-wire (TSI 1260A-T1.5), made of tungsten, had a diameter of $4 \mu \mathrm{m}$ and a length of $1.25 \mathrm{~mm}$. The sensor had a flat frequency response up to $30 \mathrm{kHz}$. Most of the PVC data were acquired with a sampling rate of $20 \mathrm{kHz}$, fast enough to resolve the fine scales. This sensor met the PVC resolution criteria. As will be shown, the small scale structure length to sensor length ratio was better than unity, 1.38 , and the maximum most probable frequency was $4 \mathrm{kHz}$, well within the response of the wire. The total sample time was 12.3 seconds. Stationarity of all parameters considered was achieved after 9.2 seconds. Estimated errors in the mean velocity are less than $1 \%$.

For the secondary velocity and streamwise vorticity measurements, the X-wire probe (TSI 1241-T1.5) was used to sample the data first in the $u-v$ and then in the $\mathrm{u}-\mathrm{w}$ orientation successively. The sampling rate was 500 $\mathrm{Hz}$. The sensitivity of the $\mathrm{X}$-wire to yaw angle was tested. At a $20^{\circ}$ angle, the measured streamwise velocity overestimated the actual streamwise velocity by $1.8 \%$. The secondary velocity recorded a $4.4 \%$ increase at this angle. The $\mathrm{X}$-wires, then, were calibrated at zero yaw and zero pitch ( $X, Z$ and $X, Y$ planes) for both the $u-v$ and $\mathrm{u}$-w configurations. The data were taken only where the average flow angles were less than $20^{\circ}$, downstream of $X=1.0$.

The streamwise vorticity measurements were also conducted by a second method utilizing two $\mathrm{X}$-wires simultaneously, with one in the $u-v$ and the other in the u-w orientation. ${ }^{3}$ While a yaw calibration was abandoned with this method, much finer grid resolution was employed in the Z-direction as well as the application of a gradient correction. Except for finer details, the amplitudes and vortex core locations obtained by the two methods agreed with each other. Except for the streamwise vorticity data, all measurements presented are from the single $\mathrm{X}$-wire data.

\section{Results}

\section{Mean Flow Quantities}

The mean streamwise velocity is defined from the dimensional velocity, $U=\left(U^{*}-U_{2}\right) /\left(U_{I}-U_{2}\right)$, so that it varied from zero $(0)$ in the low speed freestream to unity (1) in the high speed freestream. Mean velocity profiles, $U(Y)$, are presented in figure 3 . These three profiles were taken in the wake region at $X=0.5$, both with the tab (lines) and without (symbol). The profile with the tab at $Z=2.0$ is identical, within experimental error, to the no tab profile. This was still essentially true at the most downstream location, $X=8.5$. Thus, the no tab profile can be thought of as a profile at $Z=\infty$, which, for the current measurement volume, is nearly identical to $Z=$ 2.0 . The profile at the tab apex, $Z=0.0$, naturally shows a much deeper wake behind the tab. This wake region persisted to $X=2.5$ at $Z=0.0$, and to $X=1.0$ for the no tab case.

A more comprehensive picture of the effect of the tab is obtained by looking at the data in cross-stream $(Y, Z)$ planes. Figure 4 shows mean velocity contour plots, $U(Y, Z)$, at four of the downstream locations. The flow is into the page, keeping with the right hand rule. The splitter plate trailing edge and the tab are depicted with a dotted line for reference. At $X=1.0$, the wake is still evident behind the tab (dashed lines indicate negative values). Except for immediately behind the tab, there is very little distortion to the flow field beyond $Z=$ 1.0 , and the upper freestream is disturbed less that the lower freestream. Further downstream, however, the mean velocity field becomes more and more distorted. Beyond $X=2.0$, the contours start to double back on themselves at the base-edge of the tab. The significance of this on the stability of the flow will be discussed later.

Additional insight is obtained from the mean secondary velocity maps. Figure 5 focuses on the plane at $X$ $=2.0$. In figure $5 \mathrm{a}$, the vertical velocity, $V$, demonstrates a strong upwash behind the tab, and a weaker downwash at the side of the tab. The spanwise velocity, $W$, in figure $5 b$ demonstrates outflow at the top of the tab and inflow below the tab. These secondary flow patterns are consistent with the flow field that would be induced by a pair of counter-rotating streamwise vortices.

Using these data and the data grid, an estimate of the mean streamwise vorticity was obtained from $\Omega_{x}=(\Delta W / \Delta Y-\Delta V / \Delta Z)$. Figure 6 shows these data. The streamwise vortex core is clearly identifiable even at the farthest measurement location. The strength is greatest initially, and decreases downstream. Moreover, the strength of the streamwise vorticity is nominally $50 \%$ of the spanwise vorticity, as estimated from $\Delta U / \Delta Y$. 
Thus, the mean flow data demonstrate that the tab sets up a pair of counter-rotating streamwise vortices which force low speed fluid up into the high speed stream at the centerline. The effect of this vortex pair on turbulence quantities, including the small scale structures, is discussed next.

\section{Turbulence Quantities}

The small scale populations corresponding to the data of figures 4 and 6 are shown in figure 7. The values presented are normalized by a fixed value at each $X$ location: the maximum of the no tab survey, $N(X, Y, Z)$, = $N_{s s}(X, Y, Z) / N_{s s_{-} \max }(X, Z=2)$. Numbers greater than one, therefore, indicate the increase due to the effect of the tab. These contours generally follow the contortions of the mean velocity. Note that the $N$ values peak in the center of the mixing layer, where the spanwise vortex core resides. Additionally, in the span, the $N$-distribution exhibits an absolute peak near the base edge of the tab. Here the population increased by $20 \%$ over the no tab case. This peak is in close proximity to the streamwise vortex core, but biased toward high speed side of the shear layer. This observation is consistent with the expectation that it is the interaction between large scale vortical structures that generates these small scale structures. ${ }^{6,7}$

The tab has additional effects on the small scale structure. To illustrate this, data at two points in the $X=$ 4.0 plane were examined in further detail. The locations are marked with cross-hairs in figure $7 \mathrm{c}$; one is at the peak small scale population, $Z=0.75$, the other is at the maximum population away from the tab at $Z=2.0$, essentially the no tab case. Comparisons of the histograms of the small scale frequency and strain rate are shown in figures $8 a$ and $b$, respectively. The peak frequency away from the tab was $3125 \mathrm{~Hz}$; at the base edge, it increased to $4167 \mathrm{~Hz}$. These numbers were confirmed by the 1-D pseudo-dissipation spectra (data not shown for brevity). The small scale strain rate, $\Delta u / \Delta x$, likewise increased from 400 to $500 \mathrm{~s}^{-1}$. Thus, the tab not only generated more small scales, but they were also made smaller and stronger.

The PVC technique filters out all but the smallest fluid mechanic scales. The fluctuating velocity (rms), on the other hand, includes all scales. The contour map of $u^{\prime}$ at $X=4.0$ is shown in figure 9a. The shape of this map is typical of all the $X$-planes, and, except for magnitude, is nearly identical to the $v^{\prime}$ and $w^{\prime}$ contour maps. In all these maps, the maximum rms value occurred at the peak of the hairpin-like distortion, and not where $N$ peaks.

The time-averaged Reynolds stresses $u^{\prime} v^{\prime}$ and $u^{\prime} w^{\prime}$, also at $X=4.0$, are presented in figures $9 \mathrm{~b}$ and c, respectively. As expected for a mixing layer, the $u^{\prime} v^{\prime}$ is negative in the shear regions. The secondary stress $u^{\prime} w^{\prime}$ is also as expected, concentrated in the cores of the streamwise vortices. ${ }^{8,15}$ The fact that the magnitude of $u^{\prime} w^{\prime}$ is of the same order as $u^{\prime} v^{\prime}$ is striking. These data reinforce the observation made earlier that the magnitude of the two vortical components, $\Omega z$ and $\Omega x$, are nominally of the same order.

Focusing on the $u^{\prime} v^{\prime}$ in figure $9 \mathrm{~b}$, it is clear that the stress is most strongly negative at the peak of the hairpin-like distortion, where the shear $(\Delta U / \Delta y)$ is greatest. Also of interest is the nonnegligible positive region coincident with the core of the streamwise vortex. This positive region in not evident before $X=2.0$, yet it persists beyond $X=8.5$; which indicates an evolutionary dynamic. Both corresponding production terms, $-u^{\prime} v^{\prime}(\partial U / \partial y)$ and $-u^{\prime} w^{\prime}(\partial U / \partial z)$, remain positive everywhere. The transfer of energy is still from the mean flow to the turbulence. For the $u^{\prime} v^{\prime}$ case, this can only be true if the mean velocity profiles $U(Y)$ also exhibit opposite gradients. This observation prompted a closer examination of the mean velocity field.

Figure 10a shows two velocity profiles at $X=4.0$; one is at $Z=0.5$, where the positive $u^{\prime} v^{\prime}$ peak occurs, and the other is at $Z=2.0$. Clearly, the tab has changed the mean velocity profile in a substantial way. Instead of the normal tanh-like profile of a 2-D mixing layer exhibited at $Z=2.0$, the tab has caused high speed fluid to add to the lower half of the shear layer. This is due to the action of the streamwise vorticity sweeping down the high speed fluid. This profile now has two additional inflection points; each of which are potentially additional sources of instability. It is interesting to note, in fact, that for the $U$-profile at $Z=0.5$, the rms-profiles peak at the main inflection point, but the $N$-profile peaks at the hump between the two new inflection points.

In an effort to gain further insight, a 2-D inviscid linear stability analysis was performed on the two profiles shown in figure 10a. The code was modified from one for an axisymmetric jet with coflow. ${ }^{14}$ The jet radius $R$ was set to $20 \mathrm{~m}$, and mode 0 was chosen. For the essentially 2-D flow field at $Z=2.0$, Rayleigh's analysis is valid. Near the tab, however, the flow field is highly 3-D. Caution, therefore, should be used in interpreting the corresponding result. For the $Z=0.5$ profile, two solutions resulted, one each corresponding to the the two inflection points having positive gradients. The negativegradient inflection point was not found to be unstable. The growth rates are compared in figure $10 \mathrm{~b}$. The main inflection point at $Z=0.5$ has nominally the same growth rate as the one from $Z=2.0$, but at a slightly higher frequency. Of greater interest is the characteristic of the second inflection point, not only is its maximum growth rate higher than the other two, but it occurs at a higher frequency as well. This suggests that in the span, as the 
tab is approached, the instability frequency undergoes a complicated transformation to one or more higher frequencies. This shift in the large scale frequencies correlates with the shift in the small scale frequencies towards higher values, depicted in figure 8a. In a similar vein, Zohar found that the most probable small scale strain rate scaled with the large scale strain rate. ${ }^{7}$ Under the tab's influence, both the large scale and the small scale length scales are reduced. The additional inflection point in the $U(Y)$ profile can be viewed to play a significant role in this process.

\section{Concluding Remarks}

The effects of the tab on the developing shear layer are summarized in figure 11. The center of the shear layer is indicated with the solid lines, and the peak concentrations of the streamwise vorticity, small scale population and $\mathrm{rms}$ velocity are also indicated. The streamwise vorticity and small scale population concentrations are not exactly aligned, but are close to each other. The small scales are consistently concentrated on the high speed side of the streamwise vortex core. This has been true in other flows studied previously. Moreover, for this flow field, the rms velocity does not predict where the dissipative eddies occur.

It is clear that the delta tab has proven effective in enhancing the generation of the fine scale eddies. The tab not only increased the small scale population locally, but the eddies were made smaller with a corresponding increase in the small scale strain rate.

We conclude by noting finally that the role of the azimuthal vorticity in the small scale structure dynamics of the present flow may also be quite significant. This notion is reinforced by the fact that the small scale population maximum coincides with the location where the azimuthal vorticity undergoes a sharp turn. The proximity of the streamwise vortex core to the region of distorted azimuthal vorticity apparently produces the higher strain rates, which lead to the higher small scale structure activity.

\section{Acknowledgments}

The authors would like to thank Dr. M. Dahl for providing and assisting with his stability analysis code, and to Mr. C. Steffen Jr. for helpful discussions.

\section{References}

1. Ho, C.-M. \& Gutmark, E., 1987, "Vortex induction and mass entrainment in a small aspect-ratio elliptic jet", JFM, 179, 383-405.
2. Hussain, F. \& Husain, H.S, 1989, "Elliptic jets, Part 1: Characteristics of unexcited and excited jets", JFM, 208, 257-320.

3. Zaman, K.B.M.Q., 1995, "Axis switching and spreading of an asymmetric jet-role of vorticity dynamics", AIAA Paper 95-0889, 33rd Aero. Sci. Mtg. \& Ex., Reno, NV, Jan 9-12.

4. Zaman, K.B.M.Q., Reeder, M.F. \& Samimy, M., 1994, "The impact of 'tab' location relative to the nozzle exit on jet distortion", Phys. Fluids. 6(2), 778-793

5. Bradbury, L.J.S., \& Khadem, A.H., 1975, "The distortion of a jet by tabs", JFM, 70, 801 .

6. Huang, L.-S. \& Ho, C.-M., 1990, "Small scale transition in a plane mixing layer", JFM, 210, 475.

7. Ho, C.-M. \& Zohar, Y., 1994, "Fine scale turbulence production and control in a mixing layer", submitted to JFM.

8. Foss, J.K., 1994, "Small scale turbulence in a plane mixing layer", $\mathrm{PhD}$ Thesis, Univ. of So. Cal.

9. Austin, T.J., 1992, "The small scale topology of an 2:1 aspect-ratio elliptic jet", $\mathrm{PhD}$ Thesis, Univ. of So. Cal.

10. Zhang, S. \& Schneider, S.P., 1994, "Molecular-m ixing measurements and turbulent structure visualizations in a round jet with tabs", AIAA Paper 94-3082.

11. Liscinsky, D.S., True, B. \& Holdeman, J.D., 1995, "Effects of initial conditions on a single jet in a crossflow", AIAA Paper 95-2998, 31st Joint Prop. Conf., San Diego, CA, Jul 10-12

12. Zaman, K.B.M.Q., McKinzie, D.J. \& Rumsey, C.L., 1991, "Control of laminar separation over airfoils by acoustic excitation", AIAA J., 26 (7), 1075-1083.

13. Zaman, K.B.M.Q., 1993, "Streamwise vorticity generation and mixing enhancement in free jets by 'delta tabs'", AIAA Paper 93-3253, Shear Flow Conf., Orlando, FL, Jul 6-9.

14. Dahl, M. D., 1994, "The aeroacoustics of supersonic coaxial jets", NASA TM 106782, Nov, 94.

15. Bell, J.H. \& Metha, R.D., 1992, "Measurements of the streamwise vortical structures in a plane mixing layer", JFM, 239, 213-248. 
PEAK-VALLEY-COUNTTin (PVC) TEChNIQUE
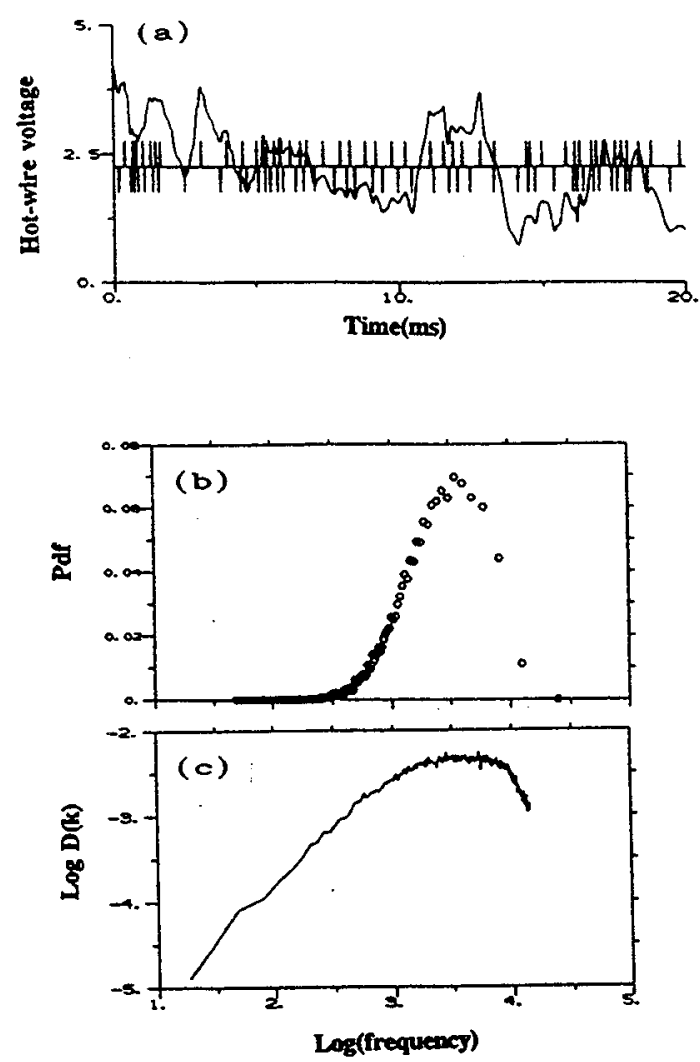

Figure 1. The Peak-Valley-Counting Technique. (a) Velocity trace and pulse train, (b) corresponding PDF of frequency (inverse period between pulses), (c) corresponding 1-D pseudo-dissipation spectrum.

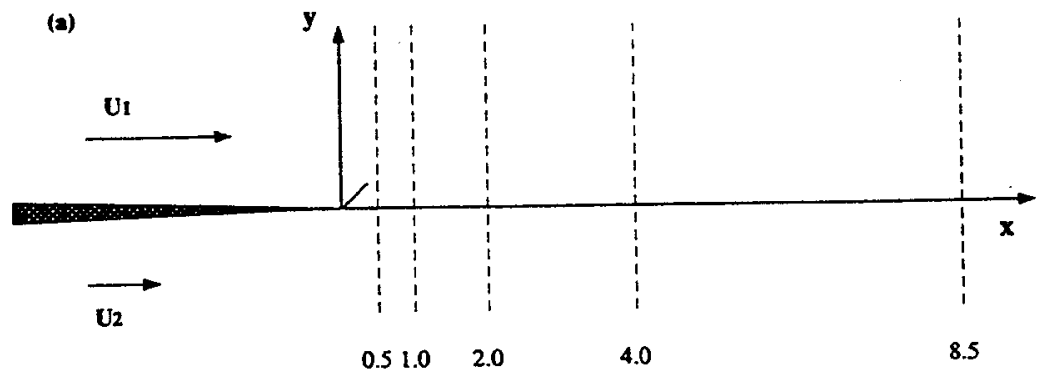

(b)

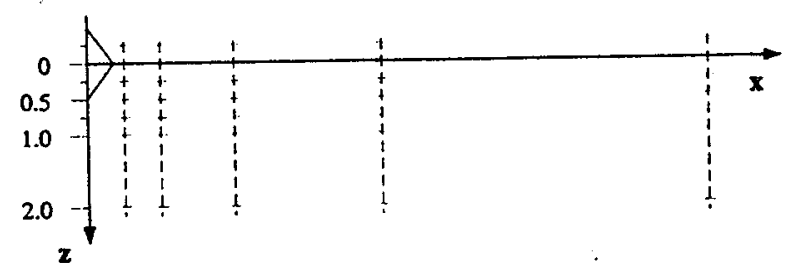

Figure 2. Trailing edge and delta tab schematic including data planes. (a) side view, (b) plan view. 


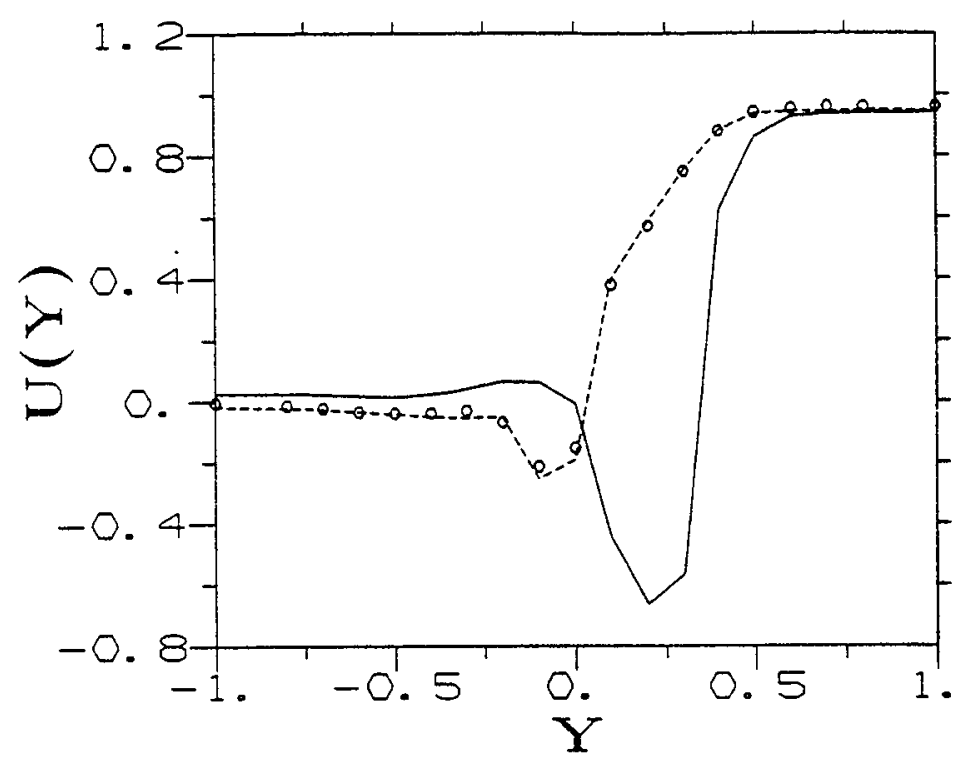

Figure 3. Velocity Profiles $U(Y)$ at $X=0.5$ : 0 , no tab; - - $Z=2.0 ;-, Z=0.5$.
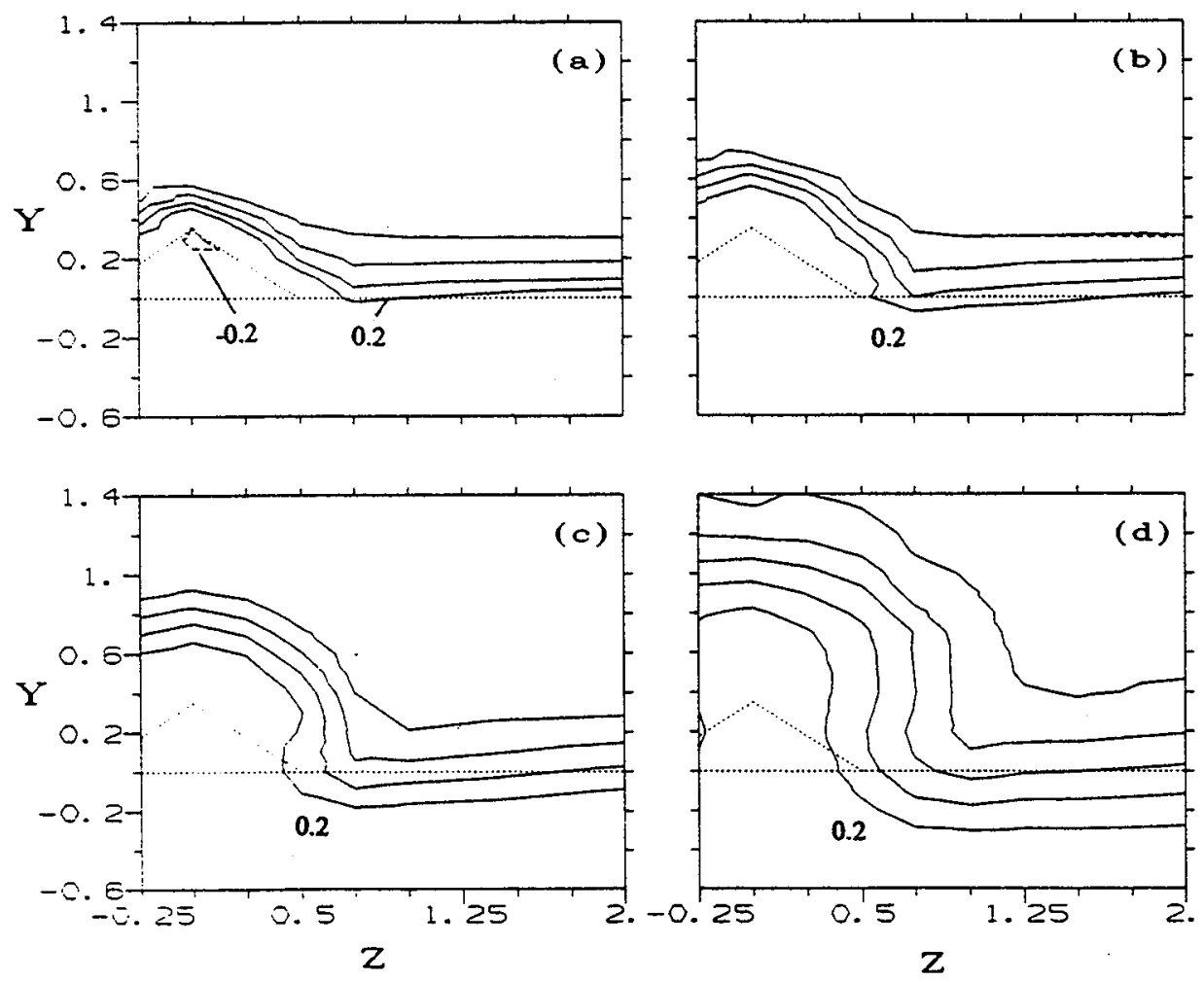

Figure 4. Velocity contours $U(Y, Z)$ at $X$ locations (a) 1.0 , (b) 2.0, (c) 4.0, (d) 8.5. Dotted line is projection of trailing edge and delta tab. Contour increments are 0.2 and start at 0.2 . 

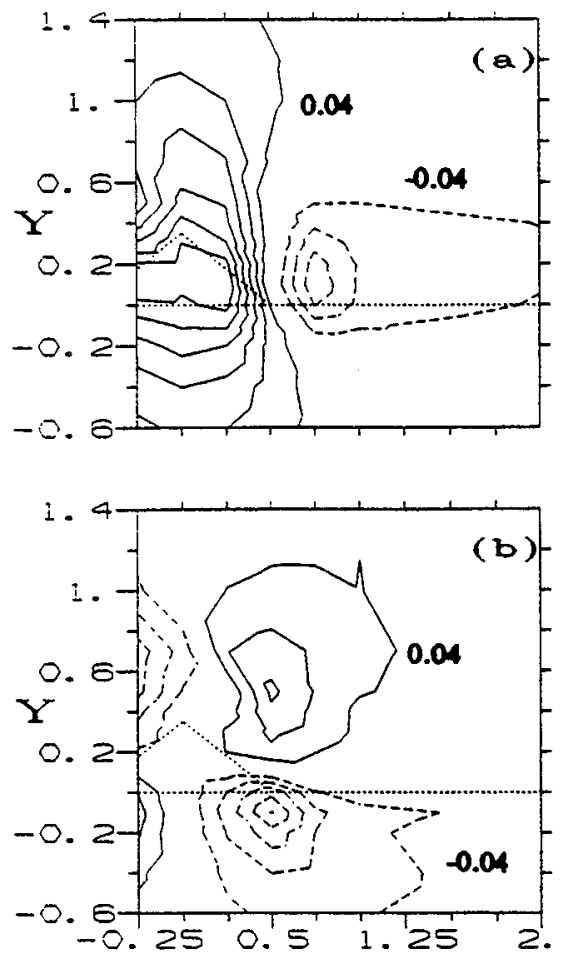

Z

Figure 5. Secondary velocity contours at $X=2.0$. (a) $V(Y, Z)$, (b) $W(Y, Z)$. Contour increments are 0.04 and start at -0.16 .
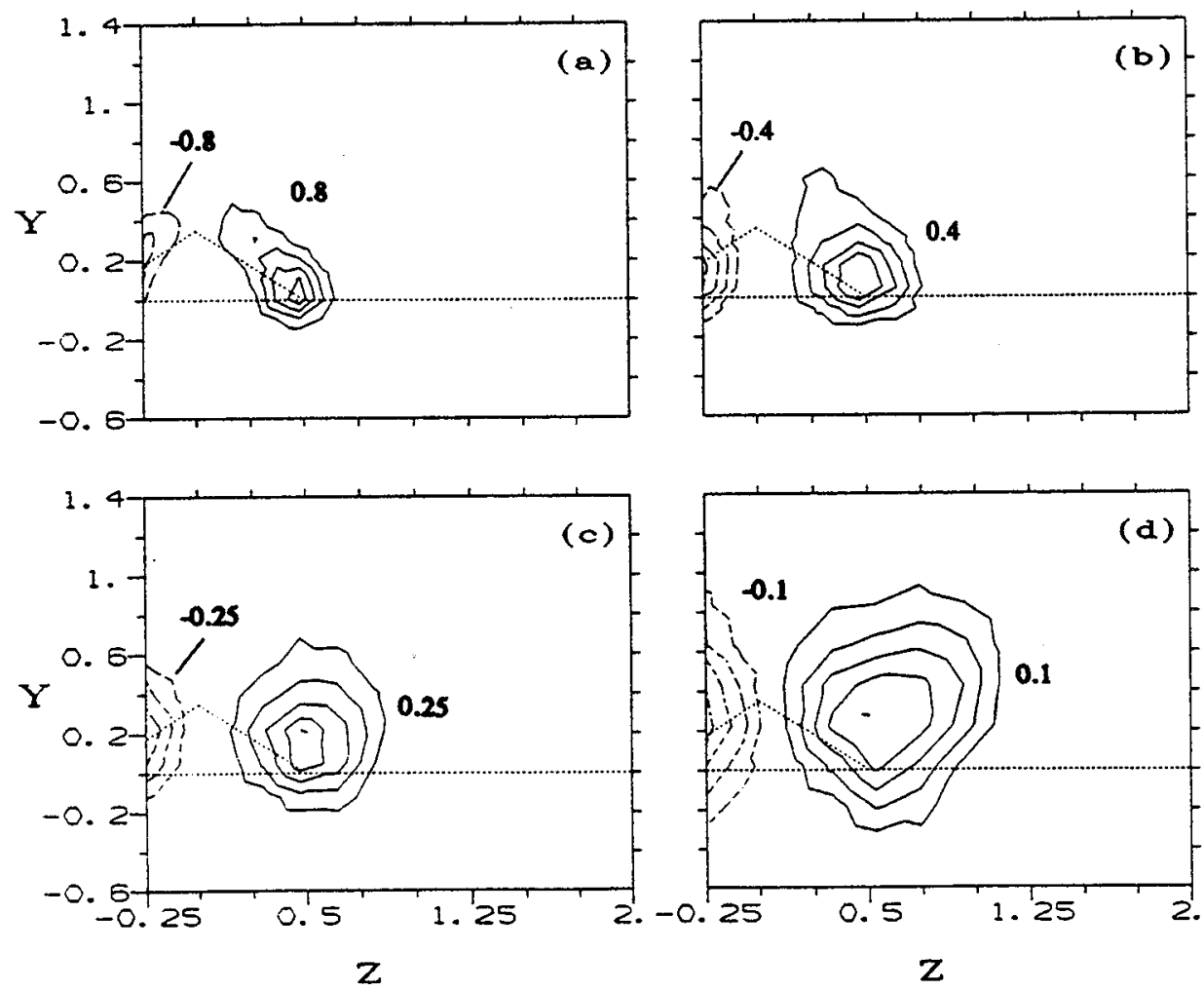

Figure 6. Streamwise vorticity contours $\Omega_{x}(Y, Z)$ at same $X$ locations as in figure 4 . Contour increment and starting levels are (a) $0.8,-4.0$; (b) $0.4,-2.0$; (c) $0.25,-1.25$; (d) $0.1,-0.5$. 

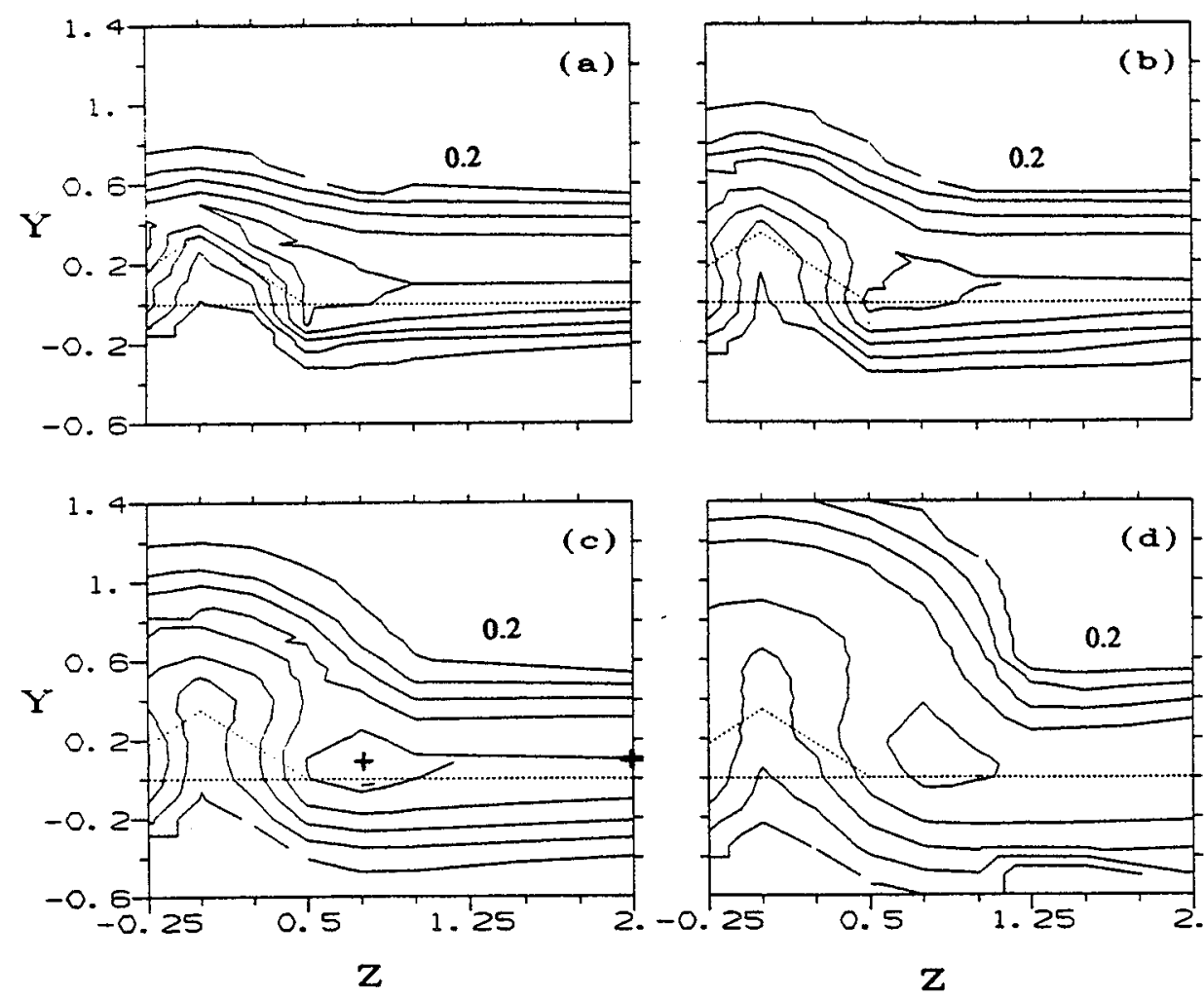

Figure 7. Small scale populations $N(Y, Z)$ at same $X$ locations as in figure 4. Contour increments are 0.2 and start at 0.2 .
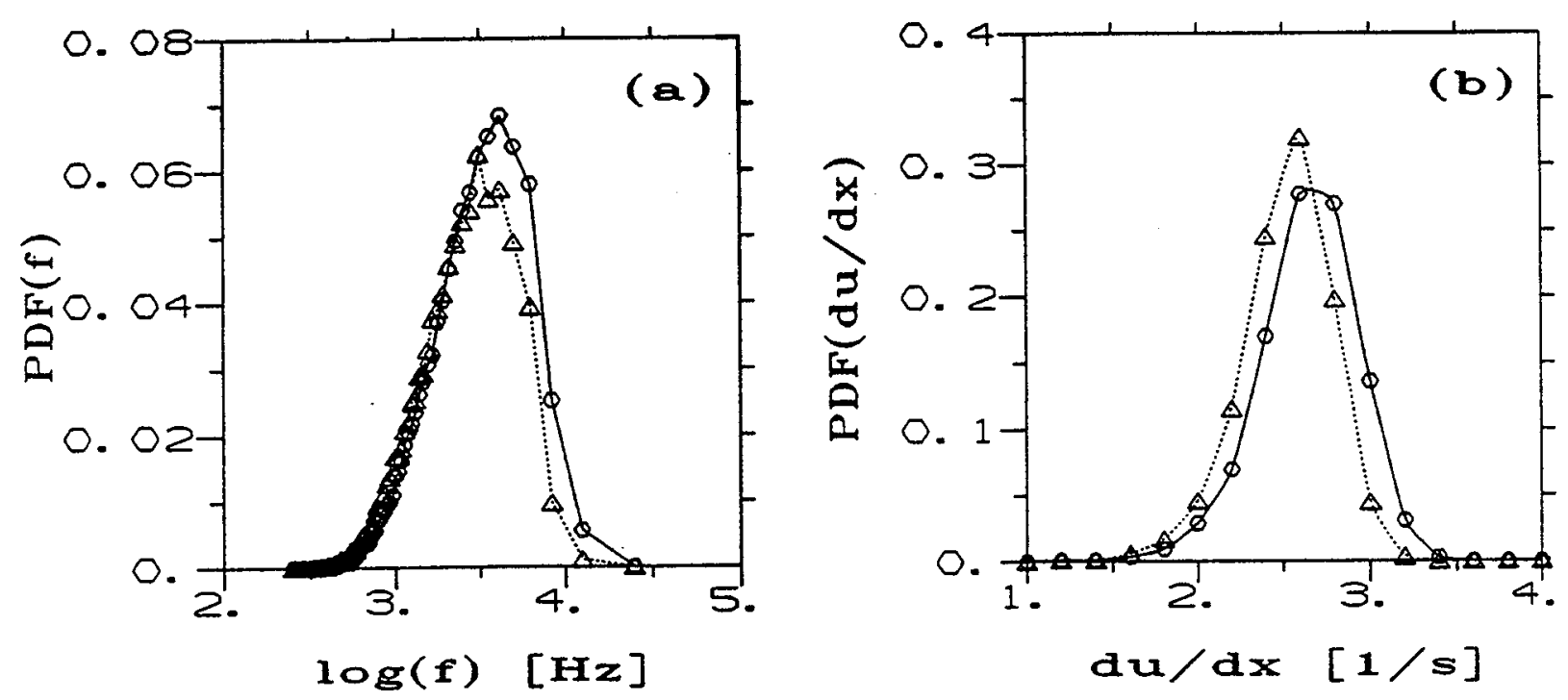

Figure 8. Spanwise comparison of the tab effect of small scale structure characteristics at the cross-hair locations depicted in figure $7 \mathrm{c}$ : $-0-, Z=0.75 ;-\Delta-, Z=2.0$. (a) frequency PDF, (b) strain rate PDF. 

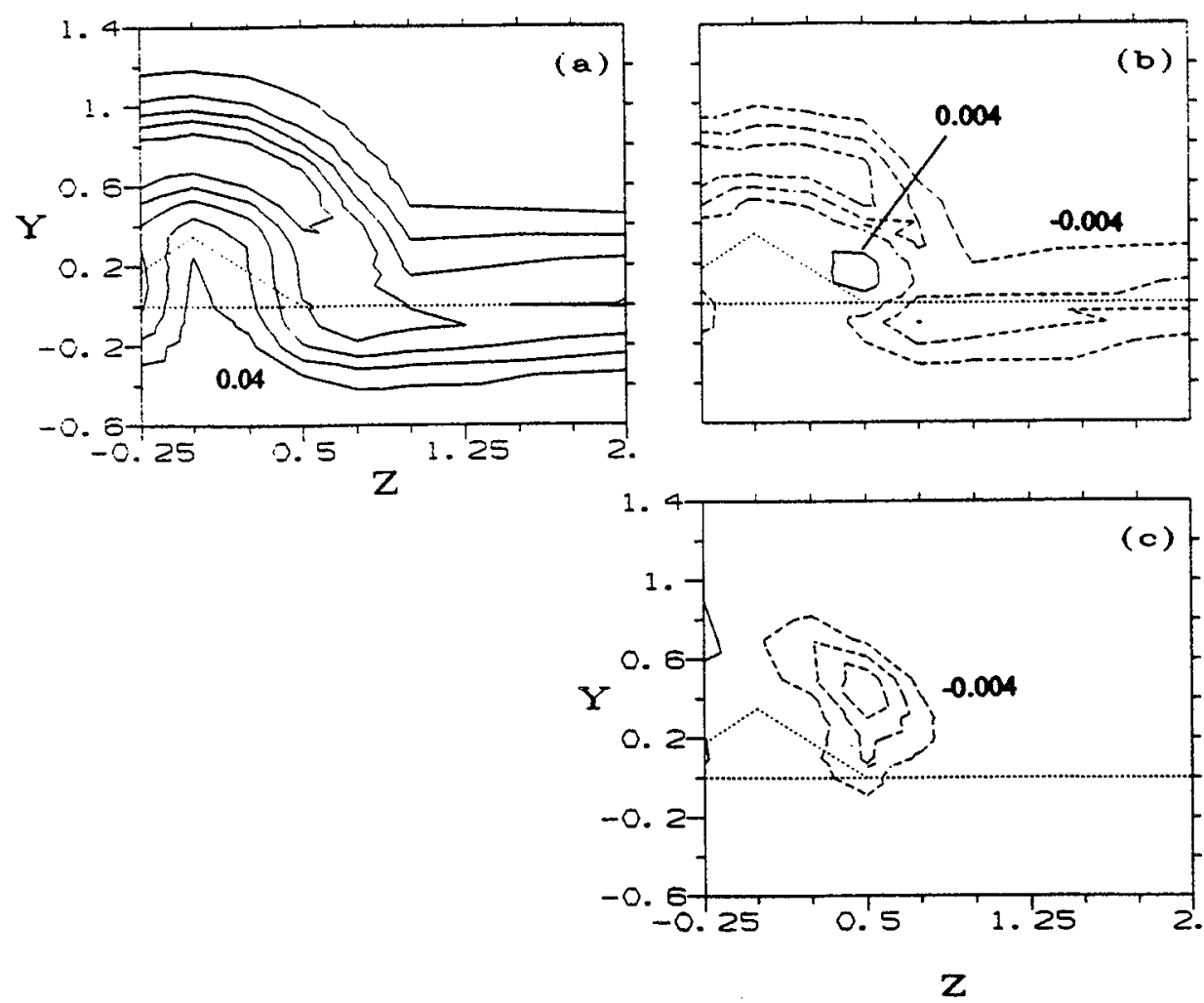

Figure 9. Turbulence characteristics at $X=4.0$. (a) $u^{\prime}(Y, Z)$, contour increment is 0.04 , start at 0.04 ; (b) $u^{\prime} v^{\prime}(Y, Z)$ and (c) $u^{\prime} w^{\prime}(Y, Z)$ contour increments are 0.004 , start at -0.016 .
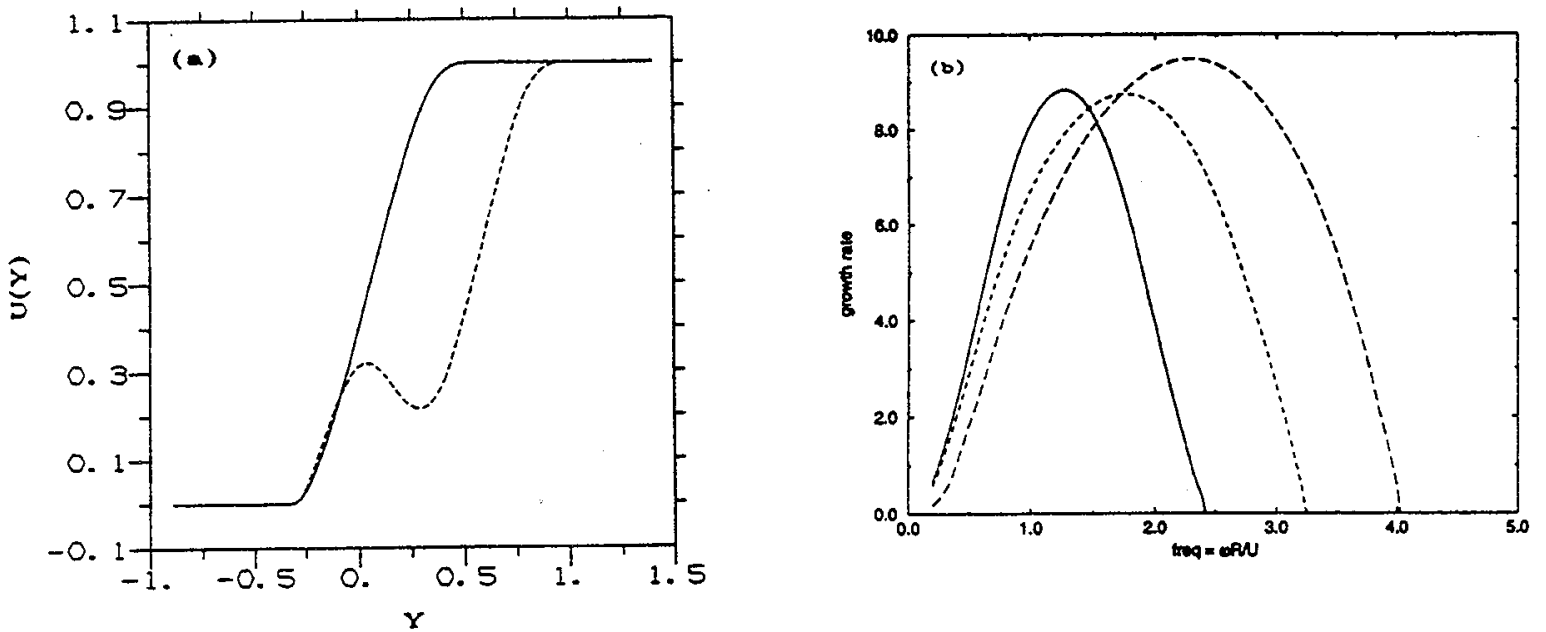

Figure 10. Comparison of tab effect on the mean velocity and its stability characteristics. (a) Mean velocity profiles $U(Y):--, Z=0.5 ;-, Z=2.0$. (b) Corresponding growth rates: $-\cdots, Z=0.5$, main inflection point;,$-- Z=0.5$, secondary inflection point;,$- Z=2.0$. 


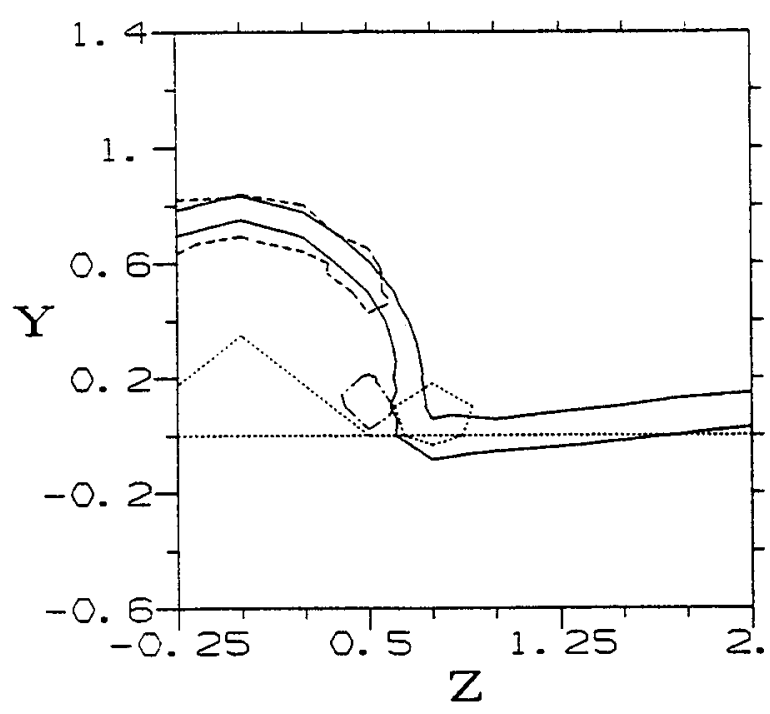

Figure 11. Summary of effects $(X=4.0)$. - $U$ (contour levels: $0.4 \& 0.6$ ); $-\cdot-, \Omega_{x}$ (contour level: $85 \%$ of $\left.\Omega_{x_{-} \max }\right) ; \cdots, N$ (contour level: $95 \%$ of $\left.N_{\max }\right) ;-\cdots, u^{\prime}\left(\right.$ contour level: $90 \%$ of $\left.u_{\max }^{\prime}\right)$ 
Public reporting burden for this collection of information is estimated to average 1 hour per response, including the time for reviewing instructions, searching existing data sources, gathering and maintaining the data needed, and completing and reviewing the collection of information. Send comments regarding this burden estimate or any other aspect of this collection of information, including suggestions for reducing this burcen, to Washington Headquaners Services, Directorate for information Operations and Reports, 1215 Jefferson Davis Highway, Suite 1204, Arlington, VA 22202-4302, and to the Oflice of Management and Budget, Paperwork Reduction Project (0704-0188), Washington. DC 20503.

\begin{tabular}{|l|l|l}
\hline 1. AGENCY USE ONLY (Leave blank) & $\begin{array}{c}\text { 2. REPORT DATE } \\
\text { January 1996 }\end{array}$ & $\begin{array}{r}\text { 3. REPORT TYPE AND DATES COVERED } \\
\text { Technical Memorandum }\end{array}$
\end{tabular}

4. TIRE AND SUBTITLE

Effect of a Delta Tab on Fine Scale Mixing in a Turbulent Two-Stream Shear

Layer

5. FUNDING NUMBERS

6. AUTHOR(S)

J.K. Foss and K.B.M.Q. Zaman

WU-537-02-22

7. PERFORMING ORGANIZATION NAME(S) AND ADDRESS(ES)

National Aeronautics and Space Administration

Lewis Research Center

Cleveland, Ohio 44135-3191

8. PERFORMING ORGANIZATION REPORT NUMBER

E-10057

9. SPONSORING/MONITORING AGENCY NAME(S) AND ADDRESS(ES)

10. SPONSORING/MONITORING AGENCY REPORT NUMBER

National Aeronautics and Space Administration

Washington, D.C. 20546-0001

NASA TM-107131

AIAA-96-0546

11. SUPPLEMENTARY NOTES

Prepared for the 34th Aerospace Sciences Meeting and Exhibit sponsored by the American Institute of Aeronautics and Astronautics,

Reno, Nevada, January 15-18, 1996. J.K. Foss, National Research Council-NASA Research Associate, at Lewis Research Center;

K.B.M.Q. Zaman, NASA Lewis Research Center. Responsible person, K.B.M.Q. Zaman, organization code 2660, (216) $433-5888$.

12a. DISTRIBUTION/AVAILABILITY STATEMENT 12b. DISTRIBUTION CODE

Unclassified -Unlimited

Subject Category 02

This publication is available from the NASA Center for Aerospace Information, (301) 621-0390.

13. ABSTRACT (Maximum 200 words)

The fine scale mixing produced by a delta tab in a shear layer has been studied experimentally. The tab was placed at the trailing edge of a splitter plate which produced a turbulent two-stream mixing layer. The tab apex tilted downstream and into the high speed stream. Hot-wire measurements in the 3-D space behind the tab detailed the three velocity components as well as the small scale population distributions. These small scale eddies, which represent the peak in the dissipation spectrum, were identified and counted using the Peak-Valley-Counting technique. It was found that the small scale populations were greater in the shear region behind the tab, with the greatest increase occurring where the shear layer underwent a sharp turn. This location was near, but not coincident, with the core of the streamwise vortex, and away from the region exhibiting maximum turbulence intensity. Moreover, the tab increased the most probable frequency and strain rate of the small scales. It made the small scales smaller and more energetic.

\begin{tabular}{|c|c|}
\hline 14. SUBJECT TERMS \\
Mixing layers; Tabs; Turbulence; Dissipation \\
\hline $\begin{array}{c}\text { 17. SECURTY CLASSIFICATION } \\
\text { OF REPORT } \\
\text { Unclassified }\end{array}$ & $\begin{array}{c}\text { 18. SECURITY CLASSIFICATION } \\
\text { OF THIS PAGE } \\
\text { Unclassified }\end{array}$ \\
\hline
\end{tabular}

\begin{tabular}{|c|c|}
\hline & $\begin{array}{c}\text { 15. NUMBER OF PAGES } \\
14\end{array}$ \\
\hline & $\begin{array}{r}\text { 16. PRICE CODE } \\
\mathrm{AO} 3\end{array}$ \\
\hline $\begin{array}{l}\text { 19. SECURITY CLASSIFICATION } \\
\text { OF ABSTRACT } \\
\text { Unclassified }\end{array}$ & 20. LIMITATION OF ABSTRACT \\
\hline
\end{tabular}


. 
National Aeronautics and

Space Administration

Lewis Research Center

21000 Brookpark Rd.

Cleveland, $\mathrm{OH}$ 44135-3191

Official Business

Penalty for Private Use $\$ 300$

POSTMASTER: If Undeliverable - Do Not Return 